\title{
Intrauterine growth restriction and later cardiovascular function
}

Fatima Crispi, MD, PhD; Francesca Crovetto, MD, PhD; Eduard Gratacós, MD, PhD

Fetal Medicine Research Center, BCNatal - Barcelona Center for Maternal-Fetal and Neonatal Medicine (Hospital Clínic and Hospital Sant Joan de Deu), ICGON, IDIBAPS, University of Barcelona, and Centre for Biomedical Research on Rare Diseases (CIBER-ER), Barcelona, Spain

\section{Funders:}

This project has been funded with support of the Erasmus + Programme of the European Union (Framework Agreement number: 2013-0040). This publication [communication] reflects the views only of the author, and the Commission cannot be held responsible for any use, which may be made of the information contained therein. Additionally, the research leading to these results has received funding form "la Caixa" Foundation under grant agreement LCF/PR/GN14/10270005, the Instituto de Salud Carlos III (INT16/00168, PI15/00130, PI14/00226, PI17/00675) integrados en el Plan Nacional de I+D+I y cofinanciados por el ISCIII-Subdirección General de Evaluación y el Fondo Europeo de Desarrollo Regional (FEDER) "Una manera de hacer Europa", Cerebra Foundation for the Brain Injured Child (Carmarthen, Wales, UK) and AGAUR 2017 SGR grant no 1531.

\section{Correspondence and reprint requests:}

\section{Fatima Crispi}

BCNatal - Barcelona Center for Maternal-Fetal and Neonatal Medicine (Hospital Clínic and Hospital Sant Joan de Deu), IDIBAPS, University of Barcelona, and Centre for Biomedical Research on Rare Diseases (CIBER-ER), Barcelona, Spain

Sabino de Arana 1, 08028 Barcelona, Spain

Telephone: +34637516976

E-mail: fcrispi@clinic.cat

Key-words: intrauterine growth restriction; fetal programming; cardiovascular disease; cardiovascular dysfunction.

Conflicts of interest statement: There are no conflicts of interest to be declared. 


\section{Abstract}

Intrauterine growth restriction is one of the most common obstetric conditions, affecting $7-10 \%$ of fetuses. Affected fetuses are actually exposed in utero to an adverse environment during the highly critical time of development and may face life-long health consequences such as increased cardiovascular risk in adulthood. Already in utero, fetuses affected by growth restriction show remodeled hearts with signs of systolic and diastolic dysfunction. Cardiovascular remodeling persist into postnatal life, from the neonatal period to adolescence, suggesting a primary fetal cardiac programming that might explain the increased cardiovascular risk later in life. In this review we summarize the current evidence on fetal cardiovascular programming in fetuses affected by growth restriction, its consequences later and possible strategies from which they could benefit to reduce their cardiovascular risk. 


\section{Introduction}

Growth and development are ongoing processes that begin at conception and continue through the remainder of our lives. However, the majority of changes occur during the prenatal period, and any interference with these early and complex processes could have consequences later in life. Fetal growth depends on several factors, such as genetics, maternal predisposition, placental state, nutrition and oxygenation: if anything is abnormal, the growth of the fetus might diverge resulting in abnormal growth and organs remodeling. This phenomenon has been called fetal programming: an adaptive response to an adverse environment in the fetal life that can cause structural, functional and metabolic changes that can persist into postnatal life increasing susceptibility to adult diseases ${ }^{1}$.

For many years cardiovascular diseases (CVD) in adulthood were thought to be determined only by genetic factors and postnatal lifestyle of individuals; however, there is now growing evidence suggesting that in most cases, CVD is triggered in early stages of life and then undergo long subclinical phase that can last decades before the first clinical symptoms appear. Already in the early 90s, the group of David Barker in Southampton, UK, established a strong and independent association between low birth weight and CVD in adulthood, including hypertension and cardiovascular mortality ${ }^{2}$. Thereafter, a large number of epidemiological and animal studies confirmed a direct link between prenatal environment and CVD in adult life, raising the concept of fetal programming ${ }^{3}$ : the complex interaction between genetics and enviroments in prenatal and early postnatal life, is determinant for the growth and development of the fetus and it defines the susceptibility to several disorders of adulthood, such as hypertension, diabetes, dyslipidemia. During prenatal life, cell proliferation and differentiation are very sensitive to any change harmful to the environment that can lead to permanent structural and functional alterations, which then may persist into the adult life. In relation to CVDs, fetal programming depends to metabolic and 
cardiovascular programming. Metabolic programming was the traditional hypothesis to explain the association between CVDs and low birth weight disease ${ }^{1,2}$ : nutrients restriction during a period of intense epigenetic programming would promote developmental pathways that suit this environment, but as in postnatal life nutrient available will be normal, this programing will lead to a higher incidence of metabolic diseases (obesity, diabetes mellitus, metabolic syndrome), which are known risk factors for CVD. However, in the last years there is a growing evidence of direct changes in the cardiovascular system that lead to a primary cardiovascular programming ${ }^{4}$.

This review presents an overview of the cardiovascular programming originated in prenatal life when fetal growth is hampered, including its later consequences in adulthood and the possible window of early preventive opportunities.

\section{Intrauterine growth restriction}

\section{Definition and etiology}

Intrauterine growth restriction (IUGR) is defined as the failure to achieve the fetal endorsed growth potential and it affects 7-10\% of all pregnancies ${ }^{5}$. Fetuses affected by IUGR have a 5- to 10folds higher risk of dying in uterus, a higher risk of perinatal morbidity, and are also at higher risk of long-term impairments, including suboptimal cognitive development and postnatal cardiovascular defects ${ }^{4}$.

Several causes have been shown to favor IUGR including smoking, maternal malnutrition, antiphospholipid syndrome, teratogen exposure, infections, genetic/structural disorders, and above all placental insufficiency, revealed by Doppler abnormalities during prenatal life. Placental insufficiency leads to fetal undernutrition, hypoxia and also pressure/volume overload of the fetal cardiovascular system. However, our knowledge of the spectrum of IUGR continues to evolve and 
the current vision is that it is a multi-phenotypic disease mainly governed by the complex interactions between genes and environment.

Different terms have been used to define a small fetus: small-for-gestational-age (SGA) and IUGR for example, have often been used interchangeably, but not all small babies are growth restricted. Obstetricians generally refer to SGA to define a constitutional small fetus, whereas IUGR usually refer to a fetus with signs of restriction, such as a very severe smallness or any Doppler abnormality ${ }^{5}$.

\section{Classification of IUGR}

Several classifications are used for IUGR, but there are two major main phenotypes according to the severity restriction ${ }^{5}$. Early-onset IUGR are fetuses with a very severe restriction, usually caused by placental insufficiency. It affects less than $1 \%$ of pregnancies and it is commonly associated with preeclampsia. The severity of the condition generally requires iatrogenic premature delivery ( $<37$ weeks) aimed at preventing intrauterine mortality and preserving maternal health.

On the other hand, in late-onset IUGR the degree of placental insufficiency is milder that reflects a less severity of restriction, allowing fetuses to be delivered near term. However, this group has also been associated with poorer perinatal outcome and long-term consequences ${ }^{4}$.

\section{Fetal cardiovascular programming}

The primary function of the heart is to provide adequate perfusion of organs; if an insult arises, the heart adapts with changes in its structure and function in order to keep its primary duty. In the initial phase of an insult, the heart undergoes a long subclinical period of dysfunction with changes in its shape, size and function and this process is called cardiac remodeling ${ }^{6}$. The intrauterine 
environment of IUGR characterized by a state of chronic hypoxia and undernutrition, together with increased placental vascular resistance, results in a combined pressure and volume overload of the fetal heart, wich induces abnormal cardiac funtion and a remodeling of its structure. Cardiac remodeling and dysfunction are thus present in IUGR fetuses as pathophysiologic mechanisms aimed at adapting to the adverse environment due to placental insufficiency. The remodeling should revert when the insult is removed, but the problem here is that it happens in prenatal life, in a very critical phase of development, when the organs are being programmed, which results that the remodeling could later persist even after the insult disappears ${ }^{3}$ underlying the fetal programming process.

\section{Fetal cardiovascular remodeling and dysfunction in IUGR}

Cardiac remodeling and dysfunction in IUGR fetuses is mostly subclinical and requires sensitive method for identification ${ }^{3}$.

Several different patterns of cardiovascular remodeling in IUGR fetuses have been described, depending on the severity of the condition and including specific structural changes of the fetal heart for most cases ${ }^{7}$. In placental insufficiency's condition, reduced oxygen and nutrients may directly affect cardiomyocytes, and at the same time the increased placental resistance to blood flow leads to an increase cardiac afterload. Consequently, the fetal heart at the beginning develops a more spherical shape to reduce wall stress and better tolerate pressure overload. In other words, the fetal heart remodels, changing to a more globular shape to better cope with the increased afterload but becomes less efficient (Figure 1). However, if the insult continues and it is also more severe, increased sphericity may not be enough: the heart becomes hypertrophic to increase contractility and decrease local wall stress. This typically occurs for the most severe cases 
(early-onset IUGR). To note, volume overload would also explain the cardiomegaly and mild pericardial effusion in this severe IUGR cases.

This profound remodeling is inevitably also associated with modifications of the cardiac function. While ejection fraction is habitually preserved until very late stages of deterioration, this occurs with a reduction in stroke volume and a concomitant compensating increase in heart rate in order to maintain cardiac output and the adequate perfusion to organs ${ }^{8}$. Accordingly, myocardial imaging techniques have permitted to demonstrate a decrease in longitudinal motion (decreased annular displacement by M-mode), and impaired relaxation. Recently, data from 2D speckle tracking imaging has also demonstrated signs of pressure overload as the presence of post-systolic shortening in the basal septal part in half of the early-onset IUGR cases $^{9}$ (figure 1, D).

Cardiovascular biomarkers in cord-blood also confirmed the cardiac dysfunction of early-onset IUGR fetuses: B-type natriuretic peptide, that is a gold standard marker of heart failure, and plasmatic troponin and heart-fatty acids-binding protein have been both reported to be increased $^{10}$. Moreover, increased cord blood levels of troponins and B-type natriuretic peptide have also been detected in some late-onset IUGR and SGA newborns ${ }^{10}$ without apparent signs of severity ${ }^{11}$.

\section{Cardiac ultrastructural changes in IUGR}

Experimental evidence suggests that IUGR is not only associated with heart shape remodeling and dysfunction, but also with profound changes in the ultrastructure of the organ. Hearts from animal models of IUGR showed more prominent and dilated coronary arteries together with a decrease in longitudinal fibers compensated by a higher proportion of circumferential fibers ${ }^{12}$. The number of cardiomyocytes was also reduced exhibiting more hypertrophic cells and altered spatial arrangement of intracellular energetic units ${ }^{12}$. The contractility machinery seemed also be altered 
with shorter sarcomere length and decreased levels of sarcomeric proteins tyrosin and myosin heavy chain. These data illustrate the profound change of the fetal heart architecture at organ, cellular and organelle levels being consistent with the postnatal persistence of cardiac remodeling induced in prenatal life.

\section{Persistence of cardiac remodeling in IUGR children}

Cardiovascular remodeling does not end in fetal life, but persists postnatally, in line with the current vision of primary fetal cardiovascular programming. Echocardiography in IUGR neonates reveals similar changes, such as more globular and less efficient hearts with reduced longitudinal motion and impaired relaxation ${ }^{8}$.

Several studies demonstrated significant cardiac changes in IUGR neonates ${ }^{13,14}$. In a cohort of newborns, Sehgal et al. described the strain (deformation from the original state) and strain rate (rate of deformation) and reported a significantly lower global deformation in SGA neonates, that reveal how the heart adapts to chronically elevated afterload by hypertrophy ${ }^{14}$.

In another cohort of 80 SGA children and 80 controls followed from fetal life up to 6 months of age $\mathrm{e}^{15}$, the same cardiovascular changes observed in the prenatal period, mainly due to a more globular cardiac shape, dilated atria, thicker myocardial walls, remained essentially unchanged at 6 months of age; these SGA newborns had also an increased mean blood pressure (BP) and aortic increased intima-media thickness (IMT). This evidence actually confirm that changes occuring during fetal life do not promptly regress after birth. Most importantly, Crispi et al. ${ }^{4}$ reported in a cohort of 80 IUGR and 120 controls evaluated at 5 years of age, more globular shape of the hearts in those children born IUGR, a reduced stroke volume and subclinical longitudinal systolic and diastolic dysfunction. Additionally, IUGR children had a higher BP and carotid IMT. All these 
previous studies support the notion that there is a direct cardiac programming in IUGR that is a main determinant of permanent postnatal cardiac and vascular differences in affected children. Vascular modifications in infants with IUGR are first described by Skilton et al. $^{16}$ : they reported a higher aortic IMT in term infants with IUGR compared to controls. The aortic or carotid IMT are good non-invasive markers of pre-clinical atherosclerosis and may serve as sentitive markers of hypertension in young IUGR children; along with cardiac remodeling, vascular impairments may also have deleterious end-organ effects ${ }^{8}$.

\section{Persistence of cardiac remodeling in IUGR adolescents and adults}

Evidence of how cardiovascular remodeling persists into later stages of life is still controversial ${ }^{17}$. Sarvari et al. ${ }^{18}$ recently reported in a cohort of preadolescents (8-12 years) similar findings as those observed in previous children cohorts: patients with a past history of IUGR have different cardiac shape with more spherical and smaller hearts, a decreased longitudinal motion and deformation and impaired relaxation. A Swedish cohort also showed smaller ventricular and vascular dimensions with preserved function in 19 young adults born with severe IUGR ${ }^{19}$. The Young Finns Study reported at an average of 31 years higher systolic BP, IMT, triglycerides and low density lipoprotein cholesterol in those born SGA ${ }^{20}$. However, in the most recent follow-up evaluation (34-49 years), there were subtle differences in heart size and lower left stroke volume, but with normal sphericity indices, diastolic function and BP in adults born SGA at term ${ }^{21}$. Similarly, The Enigma study, a long-term follow-up study of 882 young adults in UK, reported a small increase in systolic BP and central pulse pressure in those adults born SGA, but this association disappeared after adjustment for body $\operatorname{size}^{22}$. The interpretation of these studies is challenging, as multiple influences have to be taken into account during the life of these individuals and there are limitations in the accuracy of obstetric information. Future studies are 
warranted to document the dynamics of cardiovascular remodeling in adults and to better understand the link with CVD.

\section{The "second hit" theory and a window of opportunity}

Intrauterine growth restriction induces cardiovascular remodeling and programming, and individuals born IUGR should be considered at risk of CVD later in life. However, as life progresses, multiple factors seem to intervene, which as a whole could attenuate or increase the differences observed in IUGR both in utero and during early life. Whether and how other factors that intervene later in life interact with this predisposition to evolve to clinical CVD, remains a subject of research. The "second hit" hypothesis, well known in human oncology, supposes that a predisposition to a condition requires a second insult to manifest as clinical disorder. This hypothesis postulates that the cardiovascular remodeling in IUGR fetuses (first hit) confers a predisposition to CVD which neeeds to be combined with other stressors (second hit) to evolve to a clinical condition (Figure 2). This suggests that fetal programming could be improved or further worsened, depending on other exposures during life.

Children born IUGR may benefit from being followed to identify any sign of subclinical cardiac dysfunction and atherosclerosis; an excessive weight gain during infancy and early childhood, also known as catch-up growth ${ }^{23}$, should be discouraged, because it is a risk factor for later obesity and for elevated IMT. Furthermore, the early moments of life may be a unique window of opportunity (Figure 3): early interventions can have strong effects on the cardiovascular changes associated with IUGR. Observational and in-vivo studies demonstrate that brestfeeding ${ }^{24}$ and a high intake of dietary long-chain $\omega-3$ fatty acids ${ }^{25}$ improve cardiovascular remodelling in children and adolescents born IUGR. The theoretical window of opportunity for preventing strategies spans 
almost the entire life-course, but the impact of the first moments may be more relevant; preventive exposure to other risk factors, surveillance of catch-up growth, promotion of breastfeeding, healthy diet and physical activity are some of the interventions that could be recommended to this population to reduce the probability of CVD in life.

\section{Conclusions}

From a long-term perspective, IUGR probably represents the best paradigm of the impact of adverse environment during fetal life that has a consequence later in adulthood. Fetal cardiovascular remodeling in IUGR persist well into childhood and adolescence. Understanding the cardiovascular remodeling that occurs in IUGR might be useful in the monitoring and risk stratification and a more focussed intervention. Early-life preventive measures may have a strong impact in the future health of these children and merit to be carefully considered. Considering also the high prevalence of IUGR, intervention aimed at temper the detrimental effects of an altered fetal growth may have considerable effects also in terms of public health. 


\section{References}

1. Barker D. The fetal and infant origins of adult disease. BMJ 1990; 301(156): 1111.

2. D. J. Barker, C. Osmond, J. Golding, D. Kuh and MEW. Growth in utero, blood pressure in childhood and adult life, and mortality from cardiovascular disease. BMJ 1989; 298(6673): $564-567$

3. Crispi F, Miranda J, Gratacós E. Long-term cardiovascular consequences of fetal growth restriction: biology, clinical implications, and opportunities for prevention of adult disease. Am J Obstet Gynecol 2018; 218(2): S869-S879

4. Crispi F, Bijnens B, Figueras F, et al. Fetal growth restriction results in remodeled and less efficient hearts in children. Circulation 2010; 121(22): 2427-2436

5. Figueras F, Gratacós E. Update on the diagnosis and classification of fetal growth restriction and proposal of a stage-based management protocol. Fetal Diagn Ther 2014; 36(2): 86-98

6. Opie LH, Commerford PJ, Gersh BJ, Pfeffer MA. Controversies in ventricular remodelling. Lancet 2006; 367(9507): 356-367

7. Rodríguez-López $M$, Cruz-Lemini $M$, Valenzuela-Alcaraz $B$, et al. Descriptive analysis of different phenotypes of cardiac remodeling in fetal growth restriction. Ultrasound Obstet Gynecol 2017 doi:10.1002/uog.17365

8. Sehgal A, Skilton MR, Crispi F. Human fetal growth restriction: A cardiovascular journey through to adolescence. J Dev Orig Health Dis 2016; 7(6): 626-635

9. Crispi F, Bijnens B, Sepulveda-Swatson E, et al. Postsystolic shortening by myocardial deformation imaging as a sign of cardiac adaptation to pressure overload in fetal growth restriction. Circ Cardiovasc Imaging 2014; 7(5): 781-787

10. Crispi F, Hernandez-Andrade E, Pelsers MMAL, et al. Cardiac dysfunction and cell damage across clinical stages of severity in growth-restricted fetuses. Am J Obstet Gynecol 2008; 199(3)

11. Perez-Cruz M, Crispi F, Fernández MT, et al. Cord Blood Biomarkers of Cardiac Dysfunction and Damage in Term Growth-Restricted Fetuses Classified by Severity Criteria. Fetal Diagnosis and Therapy 2017 doi: 10.1159/000484315.

12. Gonzalez-Tendero A, Torre I, Garcia-Canadilla P, et al. Intrauterine growth restriction is associated with cardiac ultrastructural and gene expression changes related to the energetic metabolism in a rabbit model. Am J Physiol Circ Physiol 2013; 305(12): H1752H1760 
13. Altin H, Karaarslan S, Karataş Z, Alp H, Şap F, Baysal T. Evaluation of cardiac functions in term small for gestational age newborns with mild growth retardation: A serial conventional and tissue Doppler imaging echocardiographic study. Early Hum Dev 2012; 88(9): 757-764

14. Sehgal A, Doctor T, Menahem S. Cardiac function and arterial indices in infants born small for gestational age: analysis by speckle tracking. Acta Paediatr 2014; 103(2): e49-54

15. Cruz-Lemini M, Crispi F, Valenzuela-Alcaraz B, et al. Fetal cardiovascular remodelling persists at 6 months of life in infants with intrauterine growth restriction. Ultrasound Obstet Gynecol 2016; 48(3): 349-356

16. Skilton MR, Evans N, Griffiths KA, Harmer JA, Celermajer DS. Aortic wall thickness in newborns with intrauterine growth restriction. Lancet 2005; 365(9469): 1484-1486

17. Brodszki J, Länne T, Maršál K, Ley D. Impaired vascular growth in late adolescence after intrauterine growth restriction. Circulation 2005; 111(20): 2623-2628

18. Sarvari SI, Rodriguez-Lopez M, Nuñez-Garcia M, et al. Persistence of Cardiac Remodeling in Preadolescents with Fetal Growth Restriction. Circ Cardiovasc Imaging 2017; 10(1)

19. Bjarnegård N, Morsing $E$, Cinthio $M$, Länne $T$, Brodszki J. Cardiovascular function in adulthood following intrauterine growth restriction with abnormal fetal blood flow. Ultrasound Obstet Gynecol 2013; 41(2): 177-184

20. Raitakari OT, Juonala M, Rönnemaa T, et al. Cohort profile: The cardiovascular risk in young Finns study. Int J Epidemiol 2008; 37(6): 1220-1226

21. Arnott C, Skilton MR, Ruohonen S, et al. Subtle increases in heart size persist into adulthood in growth restricted babies: the Cardiovascular Risk in Young Finns Study. Open Hear 2015; 2(1): e000265

22. Miles KL, McDonnell BJ, Maki-Petaja KM, et al. The impact of birth weight on blood pressure and arterial stiffness in later life: The Enigma Study J Hypertens 2011; 29(12): 2324-31

23. Huxley RR, Shiell AW, Law CM. The role of size at birth and postnatal catch-up growth in determining systolic blood pressure: A systematic review of the literature. J Hypertens 2000; 18(7): 815-831

24. Rodriguez-Lopez M, Osorio L, Acosta R, et al. Influence of breastfeeding and postnatal nutrition on cardiovascular remodeling induced by fetal growth restriction. Pediatr Res 2016; 79(1-1): 100-106

25. Skilton MR, Ayer JG, Harmer JA, et al. Impaired Fetal Growth and Arterial Wall Thickening: A Randomized Trial of Omega-3 Supplementation. Pediatrics 2012; 129(3): e698-e703 
Figure 1. Cardiac shape in normal fetus (A) and in IUGR fetus (B). In the context of IUGR, increased placental resistance and reduced oxygen and nutrients increase cardiac afterload and as a consequence the fetal heart change its shape and become less efficient. Fetal echocardiography illustrates spherical cardiac shape (B) and post-systolic shortening (D) in IUGR.
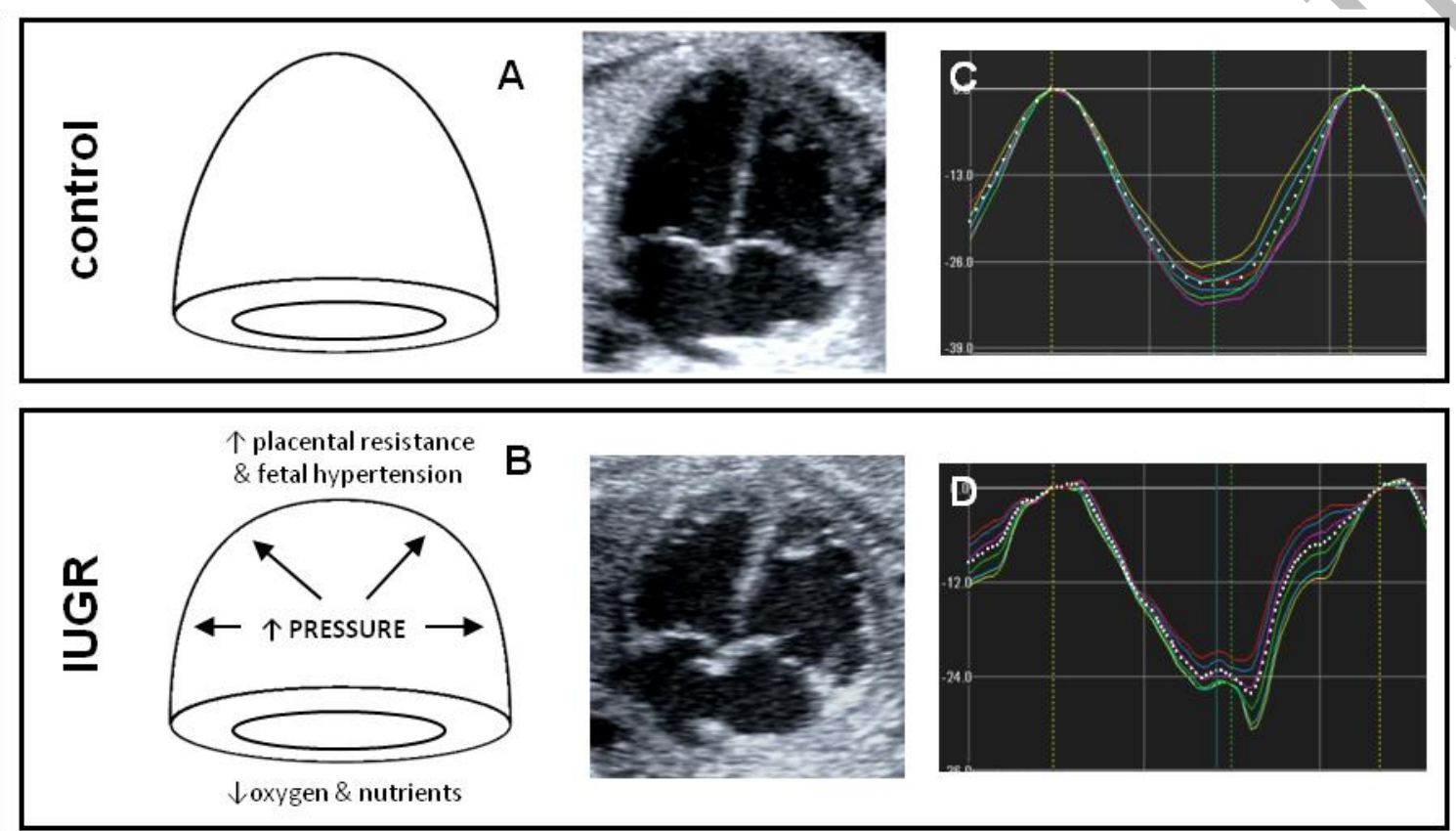
Figure 2. Hypothesis of primary fetal cardiovascular programming: IUGR induces subclinical cardiac remodeling (first hit) predisposing clinical cardiac disease, revealed after other factors (second hit) are present. This leads to the idea of prevention during window of opportunity in early stages of life.

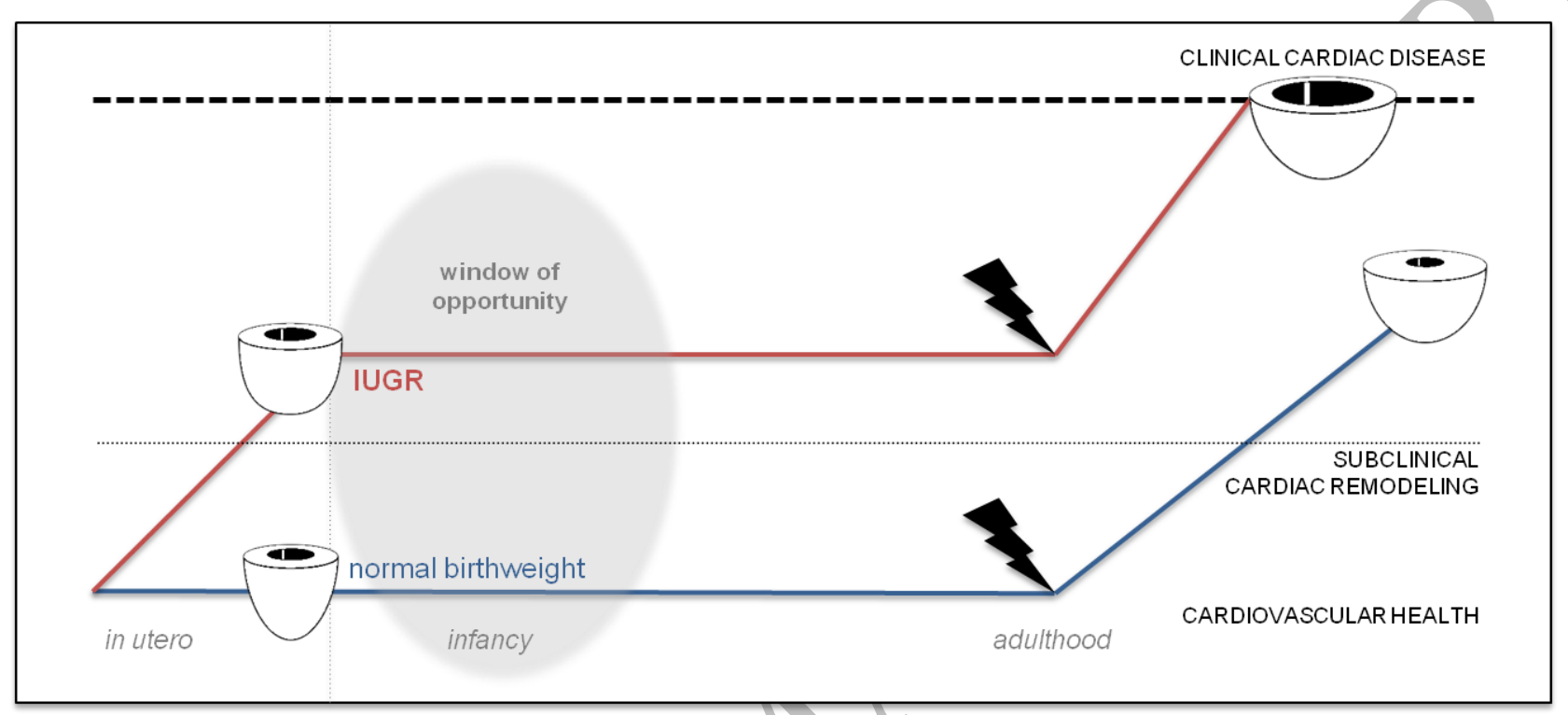


Figure 3. Fetal programming and window of opportunity in early stages of life.

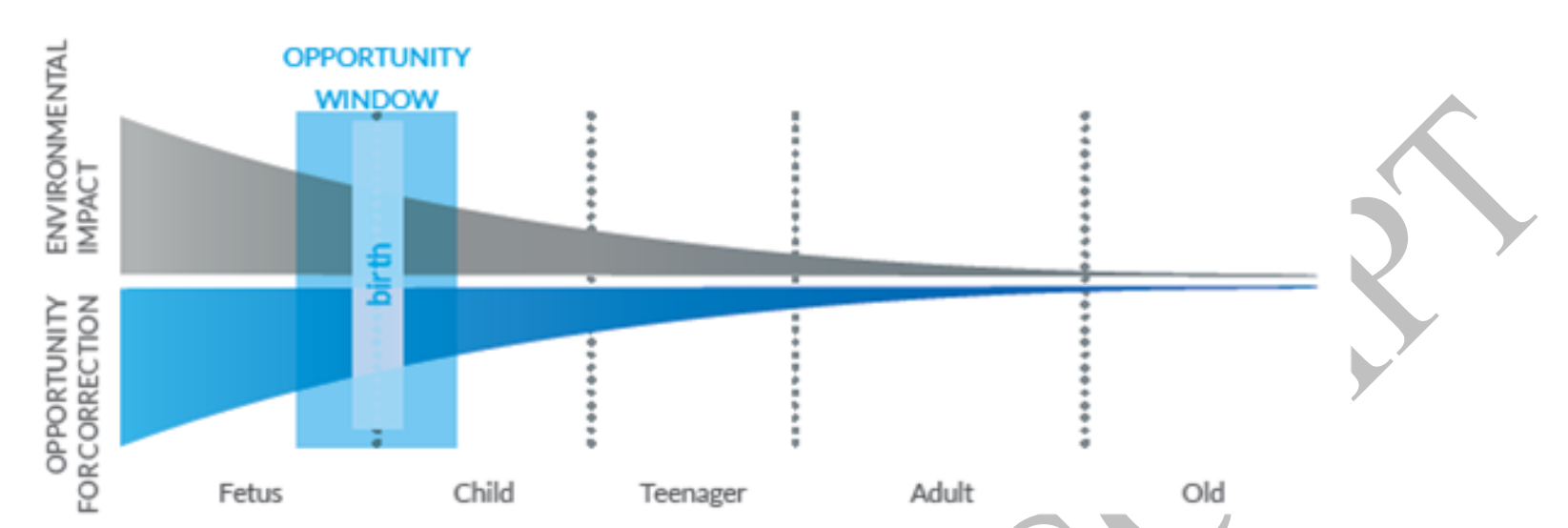

